\title{
Genome-wide association study for kernel composition and flour pasting behavior in wholemeal maize flour
}

\author{
Mara Lisa Alves ${ }^{1 *} \mathbb{D}$, Bruna Carbas², Daniel Gaspar ${ }^{3}$, Manuel Paulo $^{3}$, Cláudia Brites ${ }^{3}$, Pedro Mendes-Moreira ${ }^{3}$, \\ Carla Moita Brites ${ }^{2}$, Marcos Malosetti ${ }^{4}$, Fred van Eeuwijk ${ }^{4}$ and Maria Carlota Vaz Patto ${ }^{1}$
}

\begin{abstract}
Background: Maize is a crop in high demand for food purposes and consumers worldwide are increasingly concerned with food quality. However, breeding for improved quality is a complex task and therefore developing tools to select for better quality products is of great importance. Kernel composition, flour pasting behavior, and flour particle size have been previously identified as crucial for maize-based food quality. In this work we carried out a genome-wide association study to identify genomic regions controlling compositional and pasting properties of maize wholemeal flour.
\end{abstract}

Results: A collection of 132 diverse inbred lines, with a considerable representation of the food used Portuguese unique germplasm, was trialed during two seasons, and harvested samples characterized for main compositional traits, flour pasting parameters and mean particle size. The collection was genotyped with the MaizeSNP50 array. SNP-trait associations were tested using a mixed linear model accounting for genetic relatedness. Fifty-seven genomic regions were identified, associated with the 11 different quality-related traits evaluated. Regions controlling multiple traits were detected and potential candidate genes identified. As an example, for two viscosity parameters that reflect the capacity of the starch to absorb water and swell, the strongest common associated region was located near the dull endosperm 1 gene that encodes a starch synthase and is determinant on the starch endosperm structure in maize.

Conclusions: This study allowed for identifying relevant regions on the maize genome affecting maize kernel composition and flour pasting behavior, candidate genes for the majority of the quality-associated genomic regions, or the most promising target regions to develop molecular tools to increase efficacy and efficiency of quality traits selection (such as "breadability") within maize breeding programs.

Keywords: Zea mays L., Nutritional quality, Pasting behavior, Portuguese maize germplasm, Bread, Candidate genes, Plant breeding

\section{Background}

Maize is one of the most important food crops worldwide, and a staple crop for large populations in Latin America, Africa, and Asia [1]. Maize breeding has primarily focused on increasing stability and grain yield potential. However, in the last decade, much effort has also been made in improving maize for animal feed and human nutrition [2]. Maize food quality depends on the

\footnotetext{
* Correspondence: mara@itqb.unl.pt

${ }^{1}$ Instituto de Tecnologia Química e Biológica António Xavier, Universidade

Nova de Lisboa, Oeiras, Portugal

Full list of author information is available at the end of the article
}

raw material composition and the way that raw material is processed, which varies greatly from country to country. For instance, in Spain or Portugal, wholemeal maize flour is still frequently used for ethnic maize leavened bread production for which the local maize populations are usually preferred [3]. When using maize kernel for baking purposes, the improvement of the end-product quality can be achieved by taking into consideration the upstream processes (e.g., harvest procedures, seed quality, pest control), but also the downstream processes (e.g., milling type, baking procedure). Central to this improvement is the cultivated maize genotype, and directly

(c) The Author(s). 2019 Open Access This article is distributed under the terms of the Creative Commons Attribution 4.0 International License (http://creativecommons.org/licenses/by/4.0/), which permits unrestricted use, distribution, and 
related to this is its kernel composition and associated technological ability for bread production ("breadability"). A better understanding of the complex genetic basis of maize kernel main components and technological/processing traits is essential for devising more efficient breeding tools to support the improvement of this crop compositional quality.

The maize kernel's high nutritional value is mainly due to its starch $(\sim 72 \%$ of the kernel dry matter), protein $(\sim$ $10 \%$ of the kernel dry matter), and fat in the form of oil ( $4 \%$ of the kernel dry matter) $[4,5]$. The interactions of these three major components and fiber in a cereal-based food system are important to functionality and quality [6-8].

Many maize mutants have been widely used to isolate genes encoding key enzymes in starch metabolism, as well as genes regulating zeins, maize primary storage proteins, synthesis and deposition $[9,10]$. Currently, high-throughput genomics and post-genomics approaches are providing new tools to better understand the genetic and biochemical networks operating during maize kernel development, contributing ultimately to its composition, and a high degree of complexity and regulation has been found [9].

Linkage mapping and association mapping studies have shown that kernel main components are controlled by many genes having complex patterns of inheritance. Examples are the works of Li et al. [11] and Li et al. [12] that carried out genome-wide association studies (GWAS) to study the genetic architecture of oil and amylose biosynthesis in maize kernels, respectively. Also the work conducted by Cook et al. [13] is to be mentioned, that used a joint-linkage mapping/GWAS approach to study the genetic architecture of kernel starch, protein, and oil content. Despite the importance of maize for food, fewer works have evaluated and identified genomic regions controlling pasting properties, contrary to what has already been described in rice $[14,15]$. Wilson et al. [14], using a candidate gene approach with maize genes from the starch biosynthesis pathway, identified haplotypes of brittle endosperm2 (bt2), shrunken1 (sh1), and shrunken $2(\operatorname{sh} 2)$ that were associated with several kernel compositional traits, and haplotypes of amylose extender1 (ae1) and sh2 that were associated with starch pasting properties.

The Portuguese maize germplasm is recognized for its high diversity $[16,17]$ and associated potential quality for food, in particular for the production of the ethnic leavened maize-based broa [17], that might be associated with their higher protein contents and lower breakdown viscosities values than the values found in commercial maize varieties [18]. However this national diversity was never properly exploited for the development of efficient tools/innovative approaches to support breeding for these complex quality traits, such as "breadability".
The present study was carried out to identify genomic regions controlling the variation of maize kernel major constituents (protein, fiber, fat, and starch content) and parameters affecting the maize flour "breadability" (starch pasting properties and flour's mean particle size) through a genome-wide association approach, using a unique association panel constituted by a collection of maize inbred lines in which a considerable amount of the unexplored Portuguese maize germplasm is present.

This will allow for the understanding of the genetic architecture of quality traits, the identification of candidate quality genes, and the development of molecular selection tools for quality-related traits difficult to select by conventional methods.

\section{Results}

Portuguese traditional maize varieties known for their high "breadability" are overall characterized by lower viscosities and higher protein content than the commercial maize varieties. The present study was carried out to identify genomic regions controlling the variation of maize kernel major constituents (protein, fiber, fat, and starch content) and parameters affecting the maize flour "breadability" (starch pasting properties and flour's mean particle size) through a genome-wide association approach. For that a collection of 132 maize inbred lines, with a significant representation of lines selected from Portuguese traditional maize populations (obtained by single seed descent), was trialed during two growing seasons. The collection lines were genotyped with the MaizeSNP50 BeadChip array and the samples harvested from each field replicate characterized for main compositional traits (protein, fiber, fat, and starch), flour pasting parameters (viscosity profiles), and mean particle size.

\section{Phenotypic variation of maize flour compositional and pasting properties traits}

Considering the data obtained across the two growing seasons, fiber (FI) and protein content (PR) appeared strongly and positively correlated with phenotypic and genetic correlations coefficients superior to 0.8 (Additional file 1: Table S1 and Additional file 2: Table S2). The high pairwise genetic correlation value $(r>0.8$, Fig. 1$)$ might indicate a common genetic basis for the phenotypic variation of these traits. Fiber content (FI) had the highest heritability value ( $h^{2}=65 \%$, across growing seasons) and setback from peak viscosity (SB2) the lowest $\left(h^{2}=31 \%\right.$, across growing seasons) (Table 1), indicative that there will be more difficulties in finding the genomic regions controlling SB2 than FI.

The analysis of the variance components was performed to access the proportion of variance attributed to differences among inbred lines and also to decide if the GWAS would be carried out using the adjusted 
A

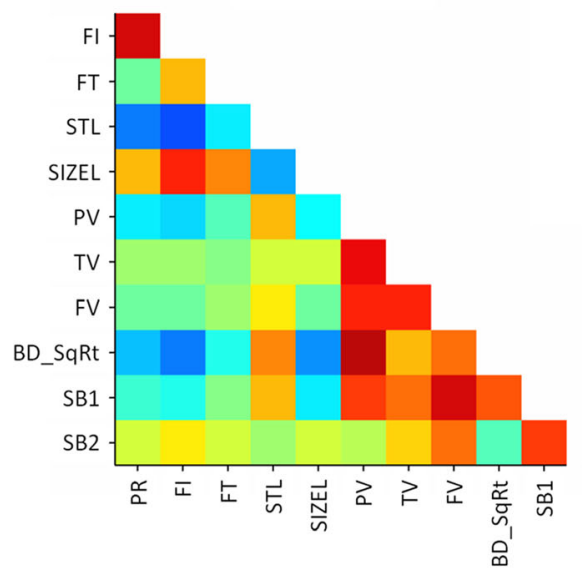

B

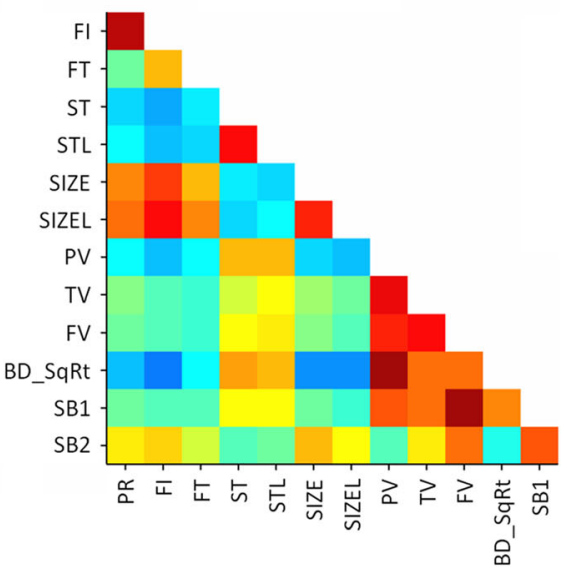

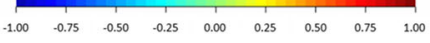

Fig. 1 Heatmaps of genetic correlations for 11 quality traits measured in 132 maize inbred lines. a 2011 growing season, b 2012 growing season. Quality traits: PR - Protein content, FI - Fiber content, FT - Fat content, ST - Starch content in non-lyophilized flour, STL - Starch content in lyophilized flour, SIZE - Mean particle size in non-lyophilized flour, SIZEL - Mean particle size in lyophilized flour, PV - Peak viscosity, TV - Trough viscosity, FV - Final viscosity, BD_SqRt - Breakdown viscosity (squared-root-transformed), SB1 - Setback from trough viscosity, and SB2 - Setback from peak viscosity

Table 1 Variance components and broad-sense heritability estimates for 11 quality traits measured in 132 maize inbred lines

\begin{tabular}{|c|c|c|c|c|c|}
\hline \multirow{2}{*}{$\begin{array}{l}\text { Trait } \\
\text { abbreviation }^{\text {a }}\end{array}$} & \multicolumn{4}{|c|}{ Variance components $^{\mathrm{b}}$} & \multirow[t]{2}{*}{$h^{2}$ heritability ${ }^{c}(\%)$} \\
\hline & $\overline{\sigma_{p}^{2}}$ & $\sigma_{g}^{2}$ & $\sigma_{g \times y}^{2}$ & $\sigma_{\text {error }}^{2}$ & \\
\hline$P R$ & 1.253 & 0.733 & 0.074 & 0.447 & 58 \\
\hline $\mathrm{FI}$ & 0.045 & 0.029 & 0.001 & 0.014 & 65 \\
\hline FT & 0.050 & 0.028 & 0.004 & 0.018 & 55 \\
\hline$S T L^{d}$ & 5.636 & 3.064 & 0.000 & 2.572 & 54 \\
\hline SIZEL $^{d}$ & 615.987 & 238.055 & 46.222 & 331.710 & 39 \\
\hline PV & $1.14 \mathrm{E} 6$ & $6.33 \mathrm{E} 5$ & 3.37E5 & $1.70 \mathrm{E} 5$ & 56 \\
\hline TV & $2.74 \mathrm{E} 5$ & $1.19 \mathrm{E} 5$ & 3.77E4 & $1.18 \mathrm{E} 5$ & 43 \\
\hline FV & $1.64 \mathrm{E} 6$ & $6.74 \mathrm{E} 5$ & $3.54 \mathrm{E} 5$ & $6.08 \mathrm{E} 5$ & 41 \\
\hline BD_SqRt ${ }^{e}$ & 167.218 & 103.016 & 36.195 & 28.007 & 62 \\
\hline SB1 & $9.53 \mathrm{E} 5$ & $3.29 \mathrm{E} 5$ & $1.58 \mathrm{E} 5$ & 4.67E5 & 34 \\
\hline SB2 & $8.22 \mathrm{E} 5$ & $2.51 \mathrm{E} 5$ & $1.89 \mathrm{E} 5$ & $3.82 \mathrm{E} 5$ & 31 \\
\hline
\end{tabular}

Quality traits measured in wholemeal maize flour from each of the 132 maize inbred lines evaluated in two growing seasons (2011 and 2012)

${ }^{a}$ Traits: PR - Protein, in \%; FI - Fiber, in \%; FT - Fat, in \%; STL - Starch, in \%; SIZEL - Mean particle size, in $\mu \mathrm{m}$; PV - Peak (maximum) viscosity, in CP; TV Trough (minimum) viscosity, in CP; FV - Final viscosity, in CP; BD_SqRt - Breakdown, in $C P$; $S B 1$ - Setback from trough viscosity, in CP; $S B 2$ - Setback from peak viscosity, in $C P$

${ }^{\mathrm{b}}$ Variance attributed to the individual terms of the statistical model: $\sigma_{p}^{2}$ corresponds to the phenotypic variance; $\sigma_{g}^{2}$ corresponds to the genotypic variance; $\sigma_{g \times y}^{2}$ corresponds to the interaction between inbred lines and growing seasons variance; $\sigma_{\text {error }}^{2}(\%)$ corresponds to the variance attributed to the block, row, column, and residual terms which altogether compose the error variance

${ }^{c} h^{2}=$ broad-sense heritability estimates obtained by fitting inbred lines as random terms in the statistical model across growing seasons

${ }^{\mathrm{d}}$ Traits values obtained from lyophilized flour

${ }^{\mathrm{e}}$ Breakdown values were squared-root transformed means across years (in case the genotypic variance component was higher than the $\mathrm{G} \times \mathrm{E}$ interaction term) or on a year's base. As shown in Table 1, the highest percentage of variance was typically due to differences between the inbred lines $\left(\sigma_{\mathrm{g}}^{2}\right)$, except for mean particle size (SIZEL), setback from trough viscosity (SB1), and setback from peak viscosity (SB2), where the error variance component was higher. The genotype-by-environment $(\mathrm{G} \times \mathrm{E})$ interaction variance component $\left(\sigma_{\mathrm{g} \times \mathrm{y}}^{2}\right)$ was higher for traits related to maize flour pasting properties (viscosity parameters). Nevertheless, and for all traits analyzed, the variance component associated with differences between inbred lines was far greater than the variance component attributed to the effect of $\mathrm{G} \times \mathrm{E}$ interaction term $\left(\sigma_{\mathrm{g}}^{2} / \sigma_{\mathrm{g} \times \mathrm{y}}^{2}>1\right)$, and so GWAS was performed using the adjusted means across years. Additional information on the collection of maize inbred lines phenotypic values (range and mean \pm standard deviation) for the quality-related traits evaluated in two growing seasons (2011 and 2012) can be found in Additional file 3: Table S3.

The maize inbred lines derived entirely from Portuguese traditional maize populations were characterized mainly by low breakdown and peak viscosity values, low starch content, and high protein, fiber, and mean particle size as can be seen in the biplot of the PCA (Fig. 2). In this analysis, the first two principal components (explaining a total of $69.74 \%$ of the variation) depicted a high diversity among the inbred lines of the association panel for the quality-related traits analyzed. 


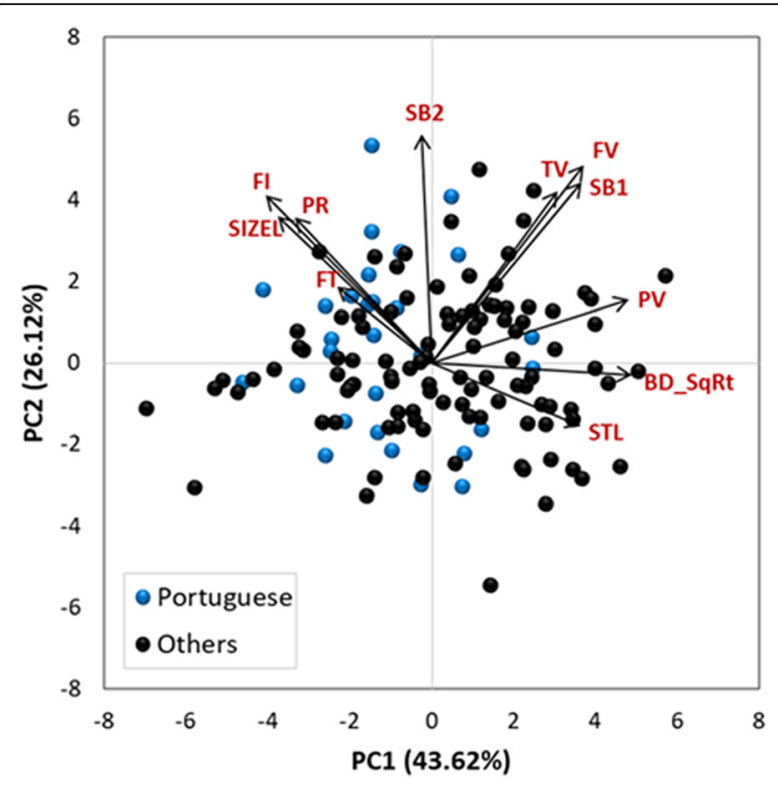

Fig. 2 Principal component analysis (PCA) biplot for 11 quality traits measured in 132 maize inbred lines. PCA based on BLUP (best linear unbiased prediction) values across growing seasons. Blue circles correspond to inbred lines selected entirely from Portuguese landraces. Quality traits: PR - percentage of protein; FI - percentage of fiber; FT - percentage of fat; STL - percentage of starch in lyophilized flour; SIZEL - mean particle size in lyophilized flour; PV - peak (maximum) viscosity; TV - trough (minimum) viscosity; FV - final viscosity; BD_SqRt - squared-root transformed values of the breakdown viscosity; SB1 - setback from trough viscosity; SB2 setback from peak viscosity

\section{Association panel genetic structure}

From the performed Eigenanalysis (Fig. 3) a wide dispersion of inbred lines was observed, with some separation according to kernel type (flint vs. dent) along the first principal component (PC1). The majority of the 29 lines selected directly from Portuguese traditional maize populations were clustered within the flint types.

The existence of strong genetic structure within the association panel can led to the identification of false positives in genome-wide association studies. In order to avoid spurious associations the GWAS was performed using a mixed linear model (MLM) and either kinship relationship ( $K$ matrix) or population structure (Eigenanalysis) was taken into account. The models that best corrects for population structure will have the inflation factor values closer to 1 . After inspecting the observed inflation factors obtained for each tested model, the mixed linear model accounting for familial relatedness ( $K$ matrix) was selected as the best model (Additional file 4: Table S4). Therefore, the results reported below concern the results obtained using this model.

For all the studied major constituents of maize kernel (protein, fiber, fat, and starch content) and all the studied parameters affecting maize flour "breadability" (starch pasting properties and flour's mean particle size), significantly associated single nucleotide polymorphism (SNP) markers were identified. In total, 72 unique SNPs were identified as being associated with at least one of the 11 quality-related traits analyzed across the two growing seasons (2011 and 2012) using a threshold $\log _{10}(P$-value $)=4$.

The 72 SNPs corresponded to 57 genomic regions (Fig. 4). While some of the genomic regions were associated with several traits, the majority of the detected genomic regions were associated with a single trait. The number of regions identified for each quality trait varied from nine for breakdown viscosity (BD_SqRt) to two regions for setback from trough viscosity (SB1). Several regions controlling multiple traits were also identified (Fig. 4), which is consistent with the strong pairwise genotypic correlations observed between some of the traits, such as peak viscosity and breakdown viscosity, final viscosity, and setback from tough viscosity or protein and fiber content (Fig. 1).

The strongest associations were found for protein content and for several viscosity parameters (Additional file 5: Table S5). Specifically, the genomic region on chromosome $1(32,314 \mathrm{~kb}$ to $32,548 \mathrm{~kb})$ was strongly associated with protein content (PR), the genomic region on chromosome $5(23,783,411 \mathrm{bp})$ was associated with setback from peak viscosity (SB2), and the genomic region on chromosome $10(60,092 \mathrm{~kb}$ to $60,351 \mathrm{~kb})$ was associated with peak viscosity (PV) and breakdown viscosity (BD_SqRt).

Five genomic regions were associated with multiple quality-related traits (Fig. 4); many of those traits were highly correlated (Fig. 1). Protein (PR) and fiber content (FI) were simultaneously associated in two different genomic regions on chromosome 1, one already described for PR, between 32,313 kilobase pairs (kb) and 32,548 kb and the other region between $267,849 \mathrm{~kb}$ and $267,886 \mathrm{~kb}$ (Fig. 5). The second region was also associated with breakdown viscosity (BD_SqRt) (Additional file 5: Table S5). Three other genomic regions were simultaneously associated with different traits related to flour's pasting properties (viscosity profiles). Namely, one genomic region associated with breakdown viscosity (BD_SqRt) and peak viscosity (PV) on chromosome 10 $(60,092 \mathrm{~kb}$ to $60,351 \mathrm{~kb})$ (Fig. 6), and two other regions associated both with setback from trough viscosity (SB1) and final viscosity (FV) on chromosome 3 (173.419 kb to $173,420 \mathrm{~kb})$ and chromosome 6 $(34,978 \mathrm{~kb}$ to $35,091 \mathrm{~kb})$ (Fig. 7).

\section{Proportion of variance explained and SNP effect size}

For all the traits, each significant SNP-trait association only explained a small portion of the observed phenotypic variance. The portion of the observed phenotypic 

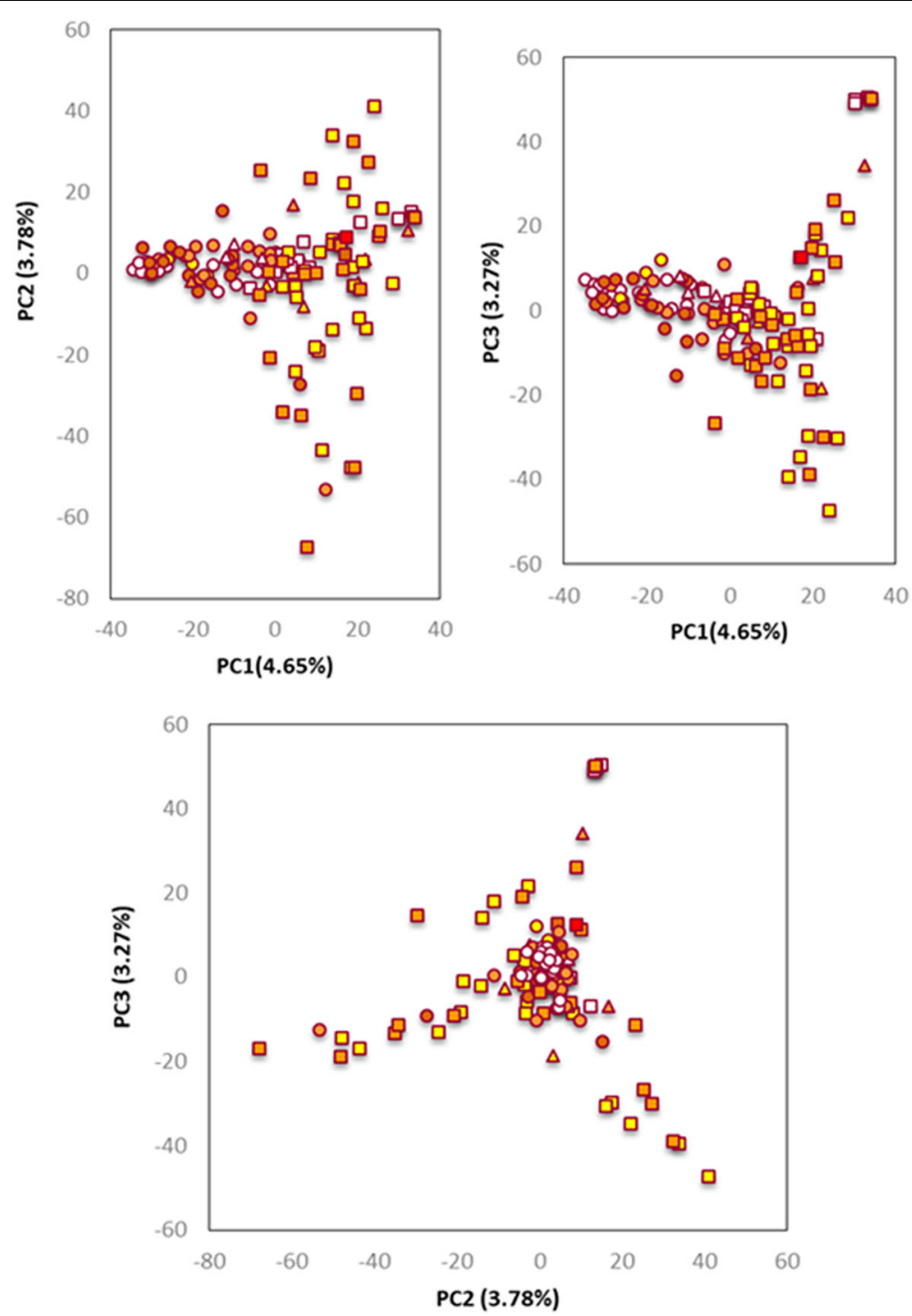

Fig. 3 First three principal component scores plots from Eigenanalysis using 1821 SNPs in 132 maize inbred lines. Inbred lines coded by endosperm type: dent (squares), flint (circles), and intermediate (triangles); and kernel color (white, yellow, yellow-orange, orange, and red). The variance explained by each principal component is given in the axis heading

variance explained by each significant SNP-trait associations was bigger in the case of setback from peak viscosity $(15.6 \%)$, and mean particle size $(14.8 \%)$ (Additional file 6: Table S6).

By inspecting the significantly associated SNP effect sizes on the traits variation we were able to identify the most promising SNPs for marker-assisted-selection (SNPs with the strongest association with the traits and with higher effect on the trait variation). Namely, for setback from peak viscosity selection, the SNP (rs131504732) which can lead to a change in $18.89 \%$ in relation to the association panel mean value would be the most logical selection choice. For peak viscosity and breakdown viscosity, the SNP (rs131765763) that can lead to a change in 12.53 and $16.30 \%$, respectively, in relation to the association panel mean value would be selected; and for protein, the choice would be the SNP (rs131232105) which can lead to a change in $4.53 \%$ in relation to the association panel mean value.

When considering the effect of the most frequent allele of the strongest SNPs associated with those quality traits and/or the SNPs that explained the biggest proportion of genetic variance on the maize inbred lines derived entirely from Portuguese populations, we observed that the frequency of the SNP variants in the Portuguese inbred lines was indeed directed toward an increase in protein, fiber, and mean particle size, and a decrease in starch, breakdown viscosity, and peak viscosity, as described before for the Portuguese derived lines. This can indicate a positive selection in the Portuguese maize 


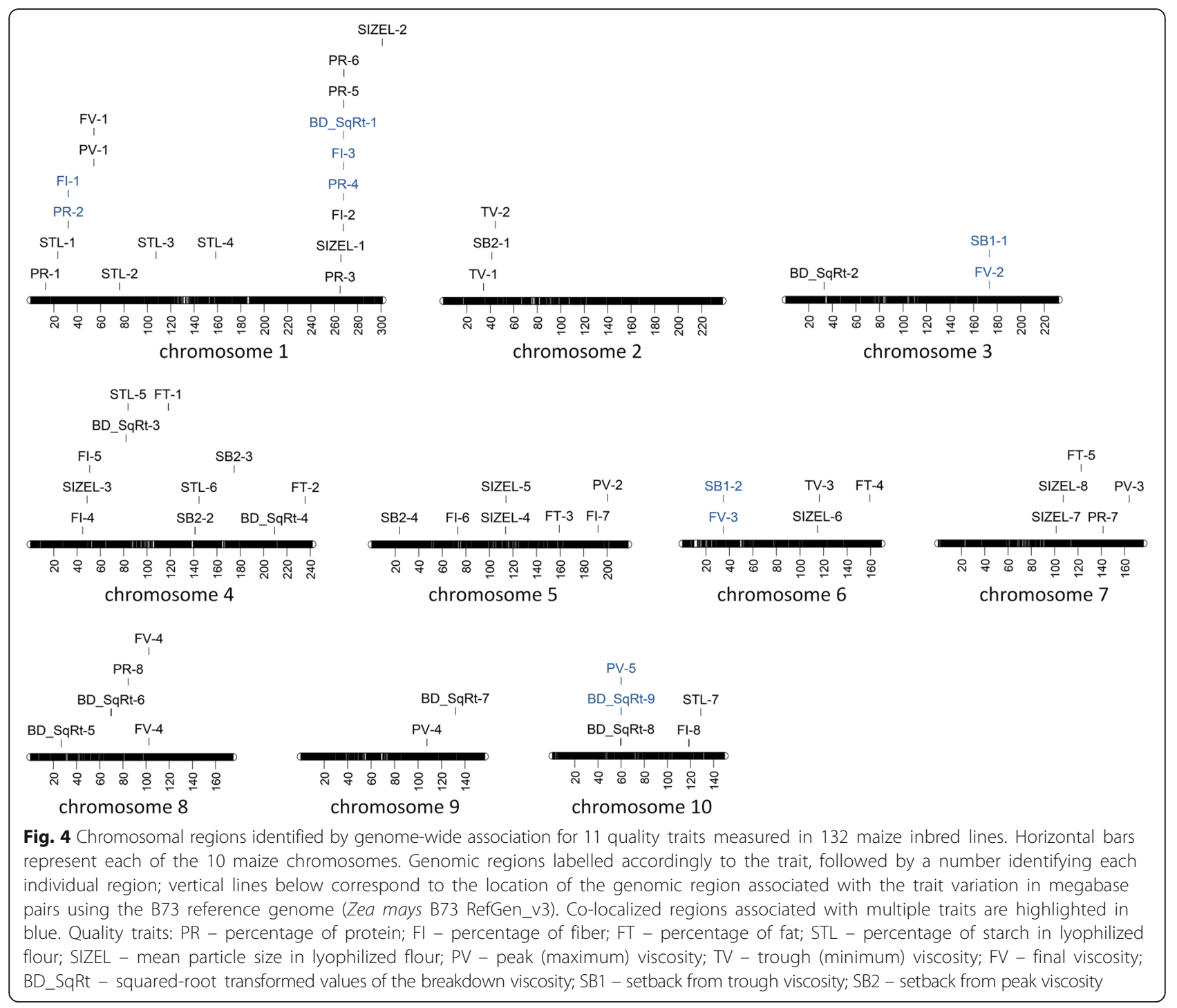

germplasm toward the presence of the favorable alleles for protein content (SNP ID rs131232105), for fiber content (SNP ID rs132587158), and mean particle size (SNP ID rs131635762), and for alleles associated with a decrease in breakdown and peak viscosities values (SNP ID rs128531960) and decrease in starch content (SNP ID rs131186983). For example, in the strongest associated SNP on chromosome 1 for protein content (SNP ID rs131232105) that the unfavorable allele was only present in $\sim 10 \%$ of the Portuguese lines.

\section{Identification of candidate genes}

We further investigated the location of genomic regions for putative candidate genes using the B73 reference genome (Zea mays B73 RefGen_v3). The degree of linkage disequilibrium in the association panel determines the size of the genomic window to be considered when looking for candidate genes as it established the degree of mapping resolution. The average linkage disequilibrium (LD) decay for the genomic regions significantly associated with the quality-related traits was for our association panel $52.23 \mathrm{~kb}$ for $\mathrm{LD} \mathrm{r}^{2}>0.2$. This value was highly variable across the genome extended to a maximum of $457 \mathrm{~kb}$ in a region of chromosome 10, spanning from $59,574 \mathrm{~kb}$ to $60,031 \mathrm{~kb}$ and identified as being associated with breakdown viscosity trait (BD_SqRt) (Additional file 5: Table S5). Using as reference the filtered gene set from the B73 RefGen_v3 assembly, a complete list of genes mapped within the significantly associated genomic regions identified in the GWAS for the 11 quality-related traits can be found in Additional file 7. A substantial proportion $(66.67 \%)$ of the SNPs significantly associated with the quality-related traits was mapped within genes (48 out of 72 SNPs significantly associated with any trait; Additional file 7: Table S7). The degree of linkage disequilibrium around the genomic regions identified by 


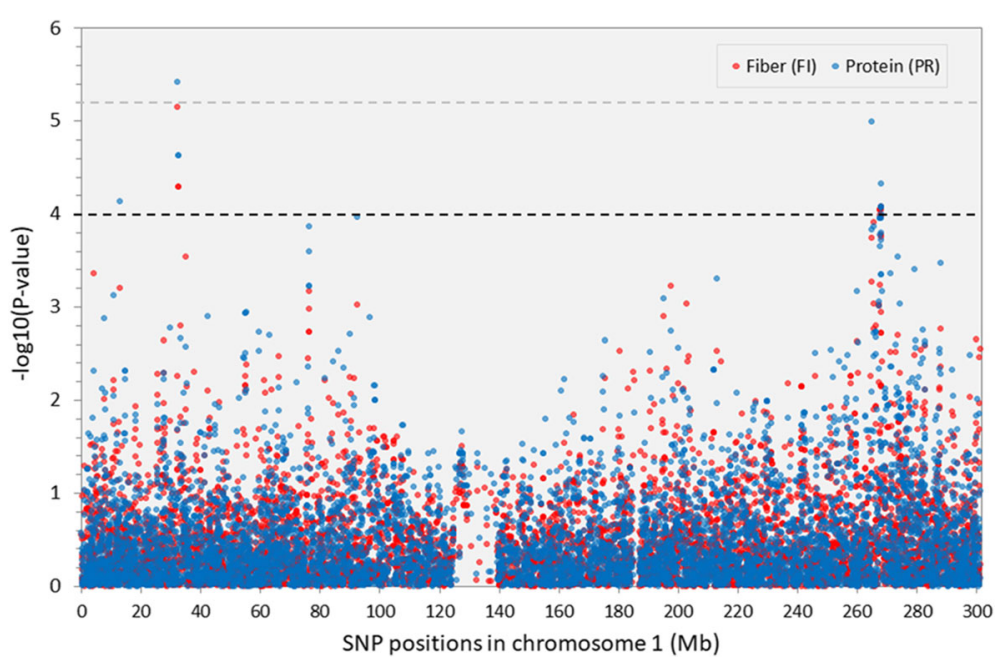

Fig. 5 Manhattan plot showing GWAS results for wholemeal maize flour protein and fiber content on chromosome 1. Y-axis shows the -log 10 $P$-values of each SNP, and $x$-axis shows their chromosomal positions. Horizontal black and grey lines represent the threshold of $P=1.00 \times 10^{-4}$, and the Bonferroni-corrected threshold of $P=6.45 \times 10^{-6}$, respectively

GWAS allowed achieving a mapping resolution to the gene level in $40.35 \%$ of the cases (LD blocks where a single gene was identified, Additional file 7: Table S7).

In the frame of this work, it was not possible to describe all candidate genes located within the associated genomic regions in detail. We therefore restrict ourselves to describe those that were (1) located within regions where the strongest significant associations were detected or (2) located within regions associated with multiple quality-related traits.

The strongest SNP-trait associations detected, corresponding to three different genomic regions, were located on chromosomes 1, 5, and 10 (SNPs highlighted in Additional file 5: Table S5). They are the genomic region on chromosome $1(32,314 \mathrm{~kb}$ to $32,548 \mathrm{~kb})$ associated with protein content (PR) and fiber (FI), the genomic region on chromosome $5(23,783,411 \mathrm{bp})$ associated with setback from peak viscosity (SB2), and the last on chromosome $10(60,092 \mathrm{~kb}$ to $60,351 \mathrm{~kb})$ associated with peak viscosity (PV) and breakdown viscosity (BD_SqRt). Some of these genomic regions harbored potential candidate genes for which we had no previous validation of their involvement with the quality-related traits analyzed. This was the case for the genomic

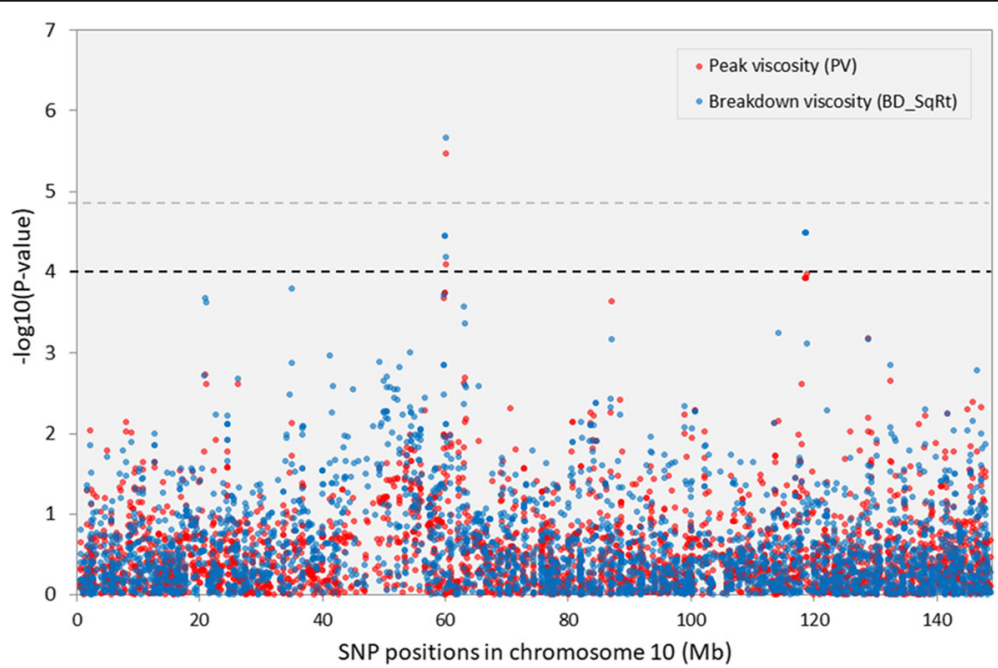

Fig. 6 Manhattan plot showing GWAS results for wholemeal maize flour peak and breakdown viscosity on chromosome 10. Y-axis shows the $-\log _{10} P$-values of each SNP, and $x$-axis shows their chromosomal positions. Horizontal black and grey lines represent the threshold of $P=1.00 \times$ $10^{-4}$, and the Bonferroni-corrected threshold of $P=1.44 \times 10^{-5}$, respectively 


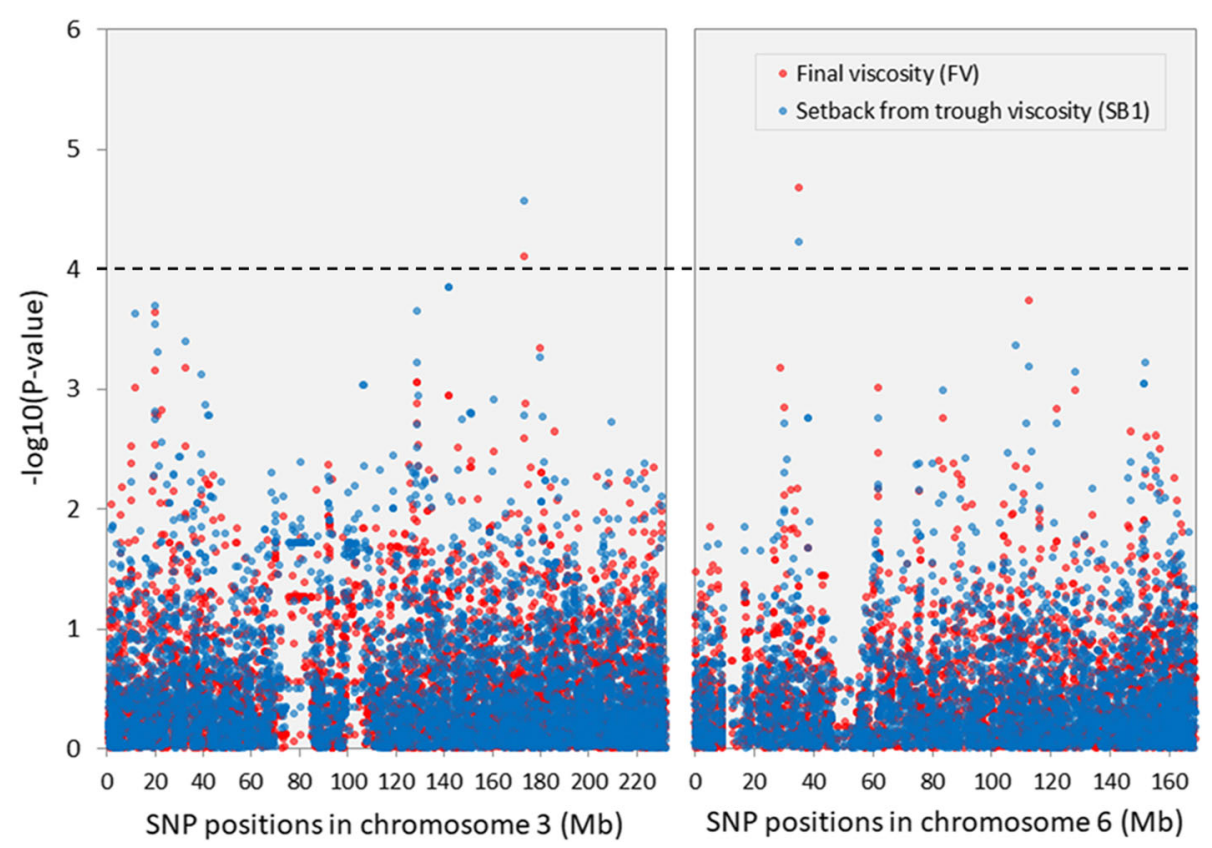

Fig. 7 Manhattan plots showing GWAS results for setback from trough viscosity and final viscosity on chromosomes 3 and 6 . Y-axis shows the $-\log _{10} P$-values of each SNP, and $x$-axis shows their chromosomal positions. The horizontal black line represents the threshold of $P=1.00 \times 10^{-4}$

regions on chromosome 1 strongly associated with PR and FI. Among the six protein-coding genes within this region, the SNP with the strongest association to both traits, rs131232105, was located within the GRMZM2G099528 gene. This gene codes for a putative endoplasmic reticulum transmembrane protein involved in the intracellular protein transport.

In the genomic region on chromosome 5, one SNP was strongly associated with setback from peak viscosity (SB2) $\left(\right.$ rs131504732, $-\log _{10}(P$-value $\left.)=5.846\right)$. This SNP was located within the GRMZM2G376743 gene, coding for a protein from the ARM repeat superfamily (PTHR33836:SF1). Finally, on chromosome 10, two SNPs were significantly associated with both peak viscosity (PV) and breakdown viscosity (BD_SqRt) (rs128531960 and rs131765763). Of those SNPs, the strongest SNP associated was rs131765763 $(-\log 10$ $(\mathrm{P}$-value $)=5.468$, for peak viscosity; and $-\log 10$ $(\mathrm{P}$-value $)=5.671$, for breakdown viscosity $)$. The SNPs associated with those two traits were not mapped within any gene. Nevertheless, considering the LD decay around those SNPs, several genes were identified within the region: GRMZM2G079777, coding for a V-type proton ATPase subunit $\mathrm{D}$ protein, involved in the phagosome and in oxidative phosphorylation; GRMZ M2G181192 ( $g l x 1$ ), coding for glyoxylase1, involved in the pyruvate metabolism; and GRMZM2G079925 and GRMZM2G005938, both coding for pentatricopeptide repeat-containing proteins.
Furthermore, candidate genes were identified for the regions associated with multiple quality-related traits. On chromosome 1, besides the region described in the previous section strongly associated with protein content, another genomic region where several candidate genes were located was associated simultaneously with breakdown viscosity (BD_SqRt), fiber (FI), and protein (PR) content. This region spanned from $267,849 \mathrm{~kb}$ to $267,886 \mathrm{~kb}$. Examples are the GRMZM2G127656 gene, which encodes a protein containing a zinc-finger domain of monoamine-oxidase A repressor R1 for fiber content, and the GRMZM2G022787 gene, which encodes for a pentatricopeptide repeat-containing protein for protein content.

Two regions were simultaneously associated with final viscosity (FV), and setback from trough viscosity (SB1), one on chromosome 3 spanning from 173,419 to $173,420 \mathrm{~kb}$ and the other on chromosome 6 spanning from $34,978 \mathrm{~kb}$ to $35,091 \mathrm{~kb}$. In the first region (chromosome 3), the significant SNP (rs131180967, $-\log _{10}$ $(P$-value $)=4.571$, for $\mathrm{FV}$, and $-\log _{10}(\mathrm{P}$-value $)=4.103$, for SB1) was mapped within the GRMZM2G452630 gene, coding for a serine hydroxyl-methyl-transferase related protein. In the second region (chromosome 6), the significant SNP (rs131176534, $-\log _{10}(\mathrm{P}$-value $)=4.675$, for $\mathrm{FV}$, and $-\log _{10}$ (P-value $)=4.224$, for SB1) was mapped within the GRMZM2G045971 gene, coding for a preprotein translocase Sec, Sec61-beta subunit protein. 


\section{Discussion}

Breeding for maize improved quality is a complex task of great economic importance presently. The development of tools, such as molecular tools, to increase efficacy and efficiency of quality selection within breeding programs is becoming crucial. In the present study and by taking advantage for the first time of the underexplored Portuguese maize germplasm selected over centuries for food uses such as bread production, we conducted a genome-wide association study, identifying 57 genomic regions and candidate genes for 11 different maize kernel constituents and parameters affecting the maize flour "breadability". These can now be used to support breeding programs for complex quality traits, difficult to select by conventional methods such as "breadability".

Starch, proteins, and lipids are the three major food components in cereal-based food products, and interactions among them in a food system are of importance to functionality and quality $[7,8]$. In a previous work we showed that Portuguese traditional maize populations considered to have high "breadability" were characterized among others by high levels of protein and fiber, and low breakdown viscosity values [19]. Additionally, the maize populations that produce better-quality bread have higher protein values and lower breakdown values when compared to commercial maize varieties [18]. Similarly, in the present work, the inbred lines derived from Portuguese maize populations were overall characterized by having a low breakdown and peak viscosity, and starch content; and a high fiber and protein content. Pasting properties of maize flour are considered important parameters in the preparation of different food products as they are related to starch swelling and gelatinization ability [20]. Besides starch, fiber and protein also influence the end-product quality. The higher protein content can potentially induce increased amounts of flour water absorption ratio and corresponding higher bread moisture. In fact, the crumb moisture has been identified [21] as a relevant attribute for consumer acceptability of maize-based bread. Fiber content can also have an impact on baked goods quality, contributing to dough viscosity, air entrapment and the improvement of loaf volume and texture [6].

The Portuguese inbred lines where mainly grouped with the lines with flint endosperm. This type of endosperm is usually associated to harder kernels. The endosperm or kernel hardness has been described as having a major impact on quality [21-23]. The size of the particles that are released from flour is directly related to the kernel hardness. Harder kernels or those richer in vitreous endosperm will yield larger particles than those that are softer [24]. With regard to the biochemical contribution to maize kernel hardness, both protein and starch composition are implicated, and specifically, the variation in zein classes has been linked to differences in hardness [23]. And both the content and composition of zeins are the key determinants to the protein quality and texture-related traits of the kernel [25].

\section{Putative pleiotropic regions associated with flour compositional and pasting properties}

Pleiotropic effects (different traits affected by the same locus/loci) can hamper breeding efforts. In this work several regions controlling multiple traits were identified, which is consistent with the observation of strong pairwise phenotypic and genotypic correlations between some of the traits (such as peak viscosity and breakdown viscosity, final viscosity, and setback from tough viscosity or protein and fiber content). This detection of genomic regions associated with multiple traits variation could be due to pleiotropic effects. However, since several genes are mapped within some of those regions, as mentioned, for instance, in Karn et al. [26], fine mapping within these regions is still required in order to properly address whether a pleiotropic gene is responsible for both traits variation or whether the traits' variation is due to two closely linked genes, and to investigate the possibility of independent selection among the correlated traits.

\section{Strong candidate genes for protein and fiber content in wholemeal maize flour}

Although for the further development of selection tools is not necessary to identify the direct causal variant, being sufficient to identify the genomic region, the identification of strong candidate gene(s) with strong evidence of direct implication on a trait variation would reinforce the overall biological results. Several of the SNP-trait associations detected in the present study were located within or near an a priori candidate gene, which strengthened and validated the usefulness of the used association panel. For instance, in the current work the allelic variation for SNP ID rs128946933 (chromosome 1 at $267,974,184 \mathrm{bp} ;-\log _{10}(P$-value $\left.)=4.002\right)$ was significantly associated with protein variation. This SNP is located within the GRMZM2G066749 gene. Recently, Chen et al. [27] demonstrated that this particular GRMZM2G066749 gene is the causative gene for dek35 mutants. The mature dek35 seeds have their protein content altered, exhibiting a significant decrease in seed dry weight and zein protein content [27]. Furthermore, the neighboring region to dek35 was previously identified by Cook et al. [13] as being associated with the maize kernel protein content.

Also the SNP with the strongest association to both protein content and fiber content (Chr1: $32,313 \mathrm{~kb}$ to $32,548 \mathrm{~kb}$; rs131232105) was located within the GRM ZM2G099528 gene. This gene codes for a putative endoplasmic reticulum transmembrane protein involved in 
the intracellular protein transport. The intracellular transport of proteins into the lumen of the endoplasmic reticulum is crucial for the protein accumulation in the kernel. Maize kernel primary storage proteins are first sequestered and accumulated in the lumen of the endoplasmic reticulum, and are afterwards directly assembled into protein bodies [25]. This region is an example of a regions identified in this work harbored putative candidate genes for which we had no previous indication of their involvement with the quality-related traits analyzed. These "novel" regions with unforeseen candidate genes, not previously described as associated to the studied traits, might be due to the present use of a different association panel, harboring different genetic variability.

Several a priori candidate genes previously identified by others as associated with maize kernel compositional traits and starch pasting properties (e.g., [13-15]) were not detected in the present study. Examples are the amylose extender1 (ae1) and shrunken2 (sh2), known also for their significant association with starch pasting properties [14]; brittle endosperm2 (bt2); shrunken1 (sh1); and dull endosperm 1 (du1), known for a significant association with kernel compositional traits. The latter, dull endosperm 1 gene (GRMZM2G141399, du1), encodes a starch synthase and is a determinant of the structure of endosperm starch in maize [28, 29]. This gene was found on chromosome 10 at a distance of approximately $46 \mathrm{~kb}$ and $564 \mathrm{~kb}$ downstream of two identified associated genomic regions with breakdown viscosity, one of the flour pasting properties studied in this work, and therefore not within the confidence intervals $(59,574-60,031 \mathrm{~kb}$ and $60,092-60,351 \mathrm{~kb}$, respectively). This observation may suggest that, at least for some regions, the rapid rate of LD decay observed in the present panel can partially explain the difficulty of detecting previously identified candidate genes named in other similar studies. Another example of a potential candidate gene for starch content, but not identified in the present study, is brittle endosperm1 gene (GRM ZM2G144081, bt1), coding a protein Brittle1 (Bt1) protein, involved in ADP-glucose transport into endosperm plastids and playing a role in starch biosynthesis [15]; or in the case of oil content in maize kernels, the acyl-CoA:diacylglycerol acyltransferase gene (GRMZ M2G169089, DGAT1-2) [13, 30]. Indeed, the genotyping platform used on the current work screened several SNPs located within all the aforementioned candidate genes. Nevertheless, no association was detected between those SNPs and the maize kernel compositional traits or starch pasting properties on the present association panel. As pointed out by [13], several factors could be responsible for differences in position and quantity of quantitative trait loci (QTLs) detected between studies, including variation in allelic frequency, mapping resolution influenced by the magnitude of linkage disequilibrium in a population, marker density, environmental effects, and QTL analysis methods.

The rapid rate of LD decay observed in the present study in the SNPs associated with the quality-related traits evaluated suggests that a higher marker density would have been beneficial in the detection of other regions putatively linked to maize flour's quality. Moreover, it is important to mention that the size of the collection of maize inbred lines used in this work most likely affected the power to detect significant markertrait associations and the subsequent identification of genomic regions controlling the analyzed traits association. As reported in Yang et al. [31], using simulation studies, a collection of 155 diverse maize lines for association mapping was suitable for studying traits mainly controlled by major QTLs. Similarly, in the present work, the collection size should have been extended in order to increase the power to detect significant markers with moderate or even minor effects on the traits.

\section{Challenges and opportunities}

This work reports the identification of 57 genomic regions associated with 11 different quality-related traits evaluated in wholemeal maize flour, highlighting candidate genes for the majority of those regions. However, novel regions, with no clear candidate genes, were also identified, which were not previously acknowledged using other germplasm collections studies.

Findings from GWAS provide valuable genetic information of trait architecture or candidate loci for subsequent validation [32]. Preliminary GWAS analysis should be complemented by statistical procedures to help prioritize GWAS results [33], such as pathway analysis of GWAS results to rank genes and pathways within a biological context. Additional follow-up analyses and experiments may even be required to pinpoint the causal genes [34]. In the present study, the SNPs strongly associated with the traits analyzed and/or the SNPs from which the allelic variant was found to contribute to the larger phenotypic effects should be prioritized as candidate genomic regions for marker development to support selection activities, especially for the quality-related traits difficult to measure/assess. The final objective is to develop the necessary expedited tools to implement routine quality selection (such as for "breadability") into maize breeding programs. As an example, by using marker-assisted selection, a few nutritional trait-associated genes or QTLs (for maize protein quality, oil content and provitamin A levels) have been recently introgressed into elite maize lines for their quality improvement [5].

Nevertheless, prior to that, the significant marker-trait associations detected in the current work need to be 
validated. Future work will concentrate on the validation of the results retrieved in this work by sequencing those regions on contrasting maize populations for the given trait. Since the actual materials used for the manufacturing of maize-based bread are the traditional maize populations, these are the ideal independent materials to proceed with the sequencing validation.

\section{Conclusions}

In this work, a genome-wide association approach was carried out to identify genomic regions controlling the variation of maize kernel major constituents (protein, fiber, fat, and starch content) and parameters affecting the maize flour "breadability" (starch pasting properties and flour's mean particle size). For that, a unique maize inbred collection containing lines partially derived from Portuguese maize populations was used given that Portuguese traditional maize populations have been developed through centuries adapted both to the local environment and food uses, in particular, for broa production.

The inbred lines derived from Portuguese maize populations were overall characterized by having a low breakdown and peak viscosity, and starch content; and a high fiber and protein content. A putative positive selection toward the presence of the favorable alleles for protein content, for fiber content, and mean particle size, and for alleles associated with a decrease in breakdown and peak viscosities values and a decrease in starch content was observed in the inbred lines derived from Portuguese traditional maize populations.

This work allowed for identifying relevant regions on the maize genome affecting maize kernel composition and flour pasting behavior, and identifying candidate genes for the majority of the flagged genomic regions. A total of 57 genomic regions were detected and candidate genes underlying the majority of those regions were identified. Moreover it was observed that the majority of the detected genomic regions were associated with a single trait. The traits for which the strongest and stable associations were found were protein, breakdown viscosity and peak viscosity. For those the strongest SNP-trait association could result in changes from more than $4 \%$ in protein content to more than $10 \%$ in the case of the viscosity parameters. Importantly, this work allowed reducing the gap towards the development of selection tools to support breeding for these complex quality traits, such as "breadability".

\section{Methods}

\section{Plant material selection}

In this work, we took advantage of the diverse inbred lines developed in the past by the extinct NUMI (Núcleo de Melhoramento de Milho) combining Portuguese germplasm with foreign (mainly US) germplasm. These inbred lines (developed by single seed descent) are currently conserved at the National Portuguese Plant Germplasm Bank (Banco Português de Germoplasma Vegetal - BPGV, Braga, Portugal). The maize inbred line collection used in this study was assembled observing a significant representation of lines selected from Portuguese traditional maize populations (29 lines) and lines with a mixed Portuguese $\times$ foreign origin (Additional file 8: Table S8).

From a total of 164 different maize inbred lines sowed on the field trials, only 132 yielded sufficient kernels to proceed with their quality analysis. Additional details on their recorded pedigree can be found in Additional file 8: Table S8. Thirty-six of the yielding lines have white kernel, further divided into 20 with flint endosperm, three intermediate and 13 with dent endosperm. The remaining 96 inbred lines have a kernel color ranging from yellow to red, further divided into 37 with flint endosperm, eight intermediate, and 51 with dent endosperm (Additional file 9: Table S9).

\section{Field characterization and experimental design}

The inbred lines were evaluated at the Coimbra site $\left(40^{\circ}\right.$ $\left.13^{\prime} 0.22^{\prime \prime} \mathrm{N}, 8^{\circ} 26^{\prime} 47.69^{\prime \prime} \mathrm{W}\right)$ in Portugal during the 2011 and 2012 growing seasons, using an organic agriculture converted field. The conversion started in 2011 and the field was considered to be fully managed under an organic agriculture system by 2012 . This site is part of the Mondego River irrigation perimeter, a very high-yielding maize area where the average maize hybrids yield is 14.5 Mg.ha ${ }^{-1}$ [35]. It is located $50 \mathrm{~km}$ from the seacoast, with an altitude of $25 \mathrm{~m}$. Its alluvial soils are characterized at $0-20 \mathrm{~cm}$ and $20-40 \mathrm{~cm}$, respectively, by a $\mathrm{pH}$ of 5.65 and 5.75; a percentage of soil with a particle size less than $0.2 \mathrm{~mm}$ diameter of 83.37 and $82.84 \%$; and an organic matter percentage of 2.91 and $2.55 \%$. Agricultural practices were similar in both growing seasons, but sowing and harvest dates differed between growing seasons. Sowing took place on April 28 and May 11 and the harvests took place on September 28 and November 6 in 2011 and 2012, respectively.

In each year, the maize inbred lines were evaluated using a randomized complete block design, with two blocks (replicates). Information on the spatial distribution of the plots was also recorded (field row and columns coordinates). Each plot consisted of two rows 7.2 $\mathrm{m}$ long $(6.4 \mathrm{~m}$ planted row plus $0.8 \mathrm{~m}$ border space between two planted rows), with an inter-row distance of $0.75 \mathrm{~m}$. Each plot was overplanted by hand and thinned at the $\mathrm{V} 7$ growth developmental stage to achieve a plant density of approximately 50,000 plants $\mathrm{ha}^{-1}$. Plots were both mechanically weeded and hand-weeded when needed and managed following common agricultural 
practices for maize in the region. Pollination was controlled within each plot. All the plots were harvested by hand. After harvest, ears were dried at $30-35^{\circ} \mathrm{C}$ in an oven (Memmert Model UFE 800, Memmert GmbH + Co. KG, Germany) until a $\sim 15 \%$ in moisture was reached. The ears were then shelled, and the kernel collected per plot basis was packed in paper bags and kept at $4{ }^{\circ} \mathrm{C}$ until further analysis.

\section{Sample preparation and phenotypic data acquisition}

A seed sample from each of the harvested plots was used for quality determinations in the two years/growing seasons. Eleven nutritional-related and technological-related traits were measured in wholemeal maize flour. Wholemeal maize flour was obtained from all the seed samples using a Falling number 3100 mill (Perten Inc., Sweden) with a $0.8 \mathrm{~mm}$ screen. In order to prevent/ minimize the enzymatic action and subsequent alteration of the flour properties, flour samples were also lyophilized using Cientificolab ${ }^{\circ}$ equipment built for pilot-scale lyophilization of food commodities. For that, each sample was individually placed in a flask (height $3.7 \mathrm{~cm}$, diameter $4.2 \mathrm{~cm}$ ) and then freeze-dried for long-term preservation.

\section{Nutritional-related traits}

Flour protein (PR), fiber (FI), and fat (FT) content were determined for each non-lyophilized sample by near-infrared reflectance (NIR) spectroscopy (Percon Inframatic 8620, Perten Inc., Sweden), with calibrations for non-lyophilized samples supplied by the manufacturer. Values for protein, fiber, and fat corresponded to the mean value of up to two technical replicates. The total starch content was determined in lyophilized (STL) (both 2011 and 2012 growing seasons) and non-lyophilized (ST) (only the 2012 growing season) samples using Fourier Transform Near-Infrared Reflectance (FT-NIR) spectroscopy (FT-NIR MPA, Bruker Optics, Germany), with calibrations for non-lyophilized samples supplied by the manufacturer. Values for total starch content obtained from the 2012 growing season lyophilized and non-lyophilized samples were further used to test whether both datasets were correlated (phenotypic correlation between datasets). Values for total starch content (non-lyophilized (ST) and lyophilized (STL) samples) corresponded to the mean value of two to four technical replicates. Protein, fiber, fat, and starch content was expressed as a percentage (\%).

\section{Technological-related traits}

The maize flour Particle Size Index (PSI) was also determined using FT-NIR spectroscopy (FT-NIR MPA, Bruker Optics, Germany). For the 2011 growing season, only the mean for particle size in lyophilized samples (SIZEL) was measured. For the 2012 growing season, both mean particle size in non-lyophilized (SIZE) and lyophilized flours (SIZEL) were determined. Values for mean particle size (non-lyophilized (SIZE) and lyophilized (SIZEL) samples) corresponded to the mean value of two to four technical replicates. The calibration models for PSI FT-NIR analysis were obtained using the particle size values measured in a subset of 30 non-lyophilized samples according to the AACC method 55-40.01:1999 [36], with a Malvern multi-channel laser light-scatter instrument (Malvern Instruments Ltd., England). Values for mean particle size obtained from lyophilized and non-lyophilized samples from the 2012 growing season were further used to test whether both datasets were correlated (phenotypic correlation between datasets). After calibration, the mean particle size volume value, or D [3, 4], retrieved from the particle size distribution, was used as an average measure of the particle size of each sample and was expressed in $\mu$ meters.

Maize flour pasting properties were evaluated by recording their viscosity profiles using a Rapid Visco Analyser (RVA) (Newport Scientific, Australia). The viscosity profiles were obtained on non-lyophilized samples according to [37] at $15 \%$ solids, using the following heating and cooling cycle set: (1) holding at $50{ }^{\circ} \mathrm{C}$ for $2 \mathrm{~min}$, (2) heating to $95^{\circ} \mathrm{C}$ for $4.5 \mathrm{~min}$, (3) holding at $95^{\circ} \mathrm{C}$ for 4.5 min, (4) cooling to $50^{\circ} \mathrm{C}$ for $4 \mathrm{~min}$, (5) holding at $50^{\circ} \mathrm{C}$ for $10 \mathrm{~min}$. The RVA paddle speed was set at $960 \mathrm{rpm}$ for the first $10 \mathrm{~s}$ of the test, after which the speed was changed to $160 \mathrm{rpm}$. The following traits were recorded: Peak (or maximum) (PV), trough (or minimum) (TV), and final (FV) viscosities. The breakdown (BD) was calculated as peak viscosity-trough viscosity, setback from trough viscosity (SB1) as final viscosity - trough viscosity, and setback from peak viscosity (SB2) as final viscosity peak viscosity. Up to two technical replicates of the viscosity profiles were taken for each sample. All the viscosity and viscosity-related traits were expressed in cPoise units.

\section{Phenotypic data analysis Phenotypic data quality control}

Quality control was performed for each year/growing season, on the data collected per genotype individually from each of the two replicated plots. Graphical inspection of residuals was used to assess normality (Q-Q plot), homogeneity of variance (residuals versus fitted values), and identify outliers. Observations were flagged for closer inspection when they exceeded 1.5 times the interquartile range, and when the standardized residuals after mixed model analysis were extreme. One of the traits (breakdown viscosity, BD) required a squared-root-transformation to stabilize the variance. All analyses were done using Breeding View software [38], available through the IBP Breeding Management System (IBP Breeding Management System Version 3.0.9, 2015). 


\section{Single/individual growing season analysis}

A phenotypic analysis was performed per single environment trial: 1) the estimates of genetic variances (and covariances between traits) and of heritability were obtained according to Oakey et al. [39], and 2) adjusted trait means for all the inbred lines were calculated.

In detail, single trait-single growing season analysis, using mixed models, was performed using the "Single trait field trial analysis" pipeline of Breeding View, selecting the model for resolvable row-column design as implemented in the software. The statistical model includes an intercept, a fixed block effect, a random row and column effects (nested within blocks), a genotypic effect (fixed or random; see the explanation that follows), and a residual. The Field trial analysis node in Breeding View performs two mixed model analyses: In the first step (Step 1) the inbred lines (genotypes) were fitted as a random term, while in the second step (Step 2) the inbred lines were fitted as a fixed term. The Step 1 model is used to obtain estimates of variance parameters. From Step 1 the heritability, as well as the best linear unbiased predictors (BLUPs), was calculated for each inbred line (and correlations between BLUPs of different traits used to obtain estimates of genetic correlations between traits). In Step 2, structural variance components (rows and column variances) are fixed to those estimated in Step 1, and by including the inbred lines as a fixed term, the best linear unbiased estimators (BLUEs) for each inbred line were produced.

\section{Multi-environment/growing seasons trial analysis}

For each quality trait, a multi-environment trial analysis was also performed to assess the consistency across growing seasons. The analysis of variance was carried out using the residual maximum likelihood (REML) variance components analysis procedure in Genstat software (Genstat ${ }^{\bullet}$ for Windows, 18th edition, [40]). The mixed model included growing seasons (fixed), maize inbred lines, and season by line interaction (fixed or random) while blocks, rows, and columns were treated as random terms and nested within growing seasons. Similar to what was already described for the single trial analysis, in the multi-environment trial analysis, BLUPs and BLUEs were calculated for each inbred line across growing seasons. BLUPs were used on principal component analysis (PCA) to assess genetic correlations between traits and BLUEs were used as input phenotypic data in the association mapping analysis, for the combined analysis across growing seasons.

\section{Genotypic data acquisition}

DNA was isolated from adult leaves from each maize inbred line using a modified CTAB procedure described in [41]. DNA quality was accessed using a $0.8 \% \mathrm{SeaKem}^{\circ}$
LE Agarose gel (Cambrex Bio Science Rockland, Inc., USA) stained with $\mathrm{SYBR}^{\circ}$ Safe (Invitrogen, USA). DNA quantification was done using a spectrophotometer Nanodrop ND-2000C (Thermo Scientific, USA). An additional step for polysaccharides removal [42] was added when the ratio $260 / 230 \mathrm{~nm}$ wavelength was inferior to 1.6 to avoid the interference of these contaminants on Single Nucleotide Polymorphism (SNP) genotyping. DNA concentration for all inbred lines was set to $50 \mathrm{ng} /$ $\mu \mathrm{l}$ and genotyped with the Illumina MaizeSNP50 BeadChip array [43]. The genotyping array procedure and alleles scoring was conducted by the genotypic service provider (TraitGenetics $\mathrm{GmbH}$, Gatersleben, Germany). This array allowed the screening of 17,520 genes (since 33,417 of the SNPs present in this array are located on 17,520 genes and 16,168 SNPs are located in intergenic regions) [43].

The position of each marker along the maize B73 reference genome was updated from the markers' coordinates available when the MaizeSNP50 BeadChip was originally designed (B73 reference genome version 1) to the coordinates in the released B73 reference genome version 3 . These coordinates were taken from the maize genome browser, via the MaizeGDB database ([44], www.maizegdb.org).

\section{Genotypic data analysis \\ Genotypic data quality control}

Genotypic data quality control was performed by removing SNP markers and inbred lines with more than $25 \%$ of missing data. SNPs called as heterozygous were set as missing data $(0.93 \%$ of the total SNP calls). Moreover, markers with a minor allele frequency (MAF) smaller than $5 \%$ were removed. After this filter, a total of 48,772 SNPs remained and were used for the association mapping analysis.

\section{Genetic structure analysis}

A subset of 1821 SNPs, evenly distributed across the genome (corresponding approximately to 1 SNP per Megabase pairs, Mb), was used to calculate principal components to study the population structure among inbred lines and to calculate the kinship matrix to estimate pairwise genetic relatedness among inbred lines as implemented in Genstat software (Genstat ${ }^{\circ}$ for Windows, 18th edition, [40]).

\section{Association mapping analysis}

Given that for all the quality-related traits under study, the variance components for genotype-by-environment $(G \times E)$ interaction $\left(\sigma_{g \times y}^{2}\right)$ were much smaller than the genotype variance component $\left(\sigma_{\mathrm{g}}^{2}\right)$, univariate association analysis was carried out using the adjusted means for field trial design (BLUEs) obtained across growing 
seasons. Genome-wide association studies were conducted with the Genstat software using the available genotypic (SNPs scored with the MaizeSNP50 BeadChip array) and quality data (11 quality-related traits) measured in 132 maize inbred lines. The Genstat software performs association mapping in the mixed model framework, fitting markers as fixed and inbred lines as random terms using REML [45].

Three different models were tested to detect significant marker-trait associations: the naïve model [Phenotype $=S N P+($ Genotype + Error $)]$, that neither accounts for population structure nor familial relatedness; a model accounting for population structure $(Q)$ using 15 principal components from PCA $[$ Phenotype $=Q+S N P$ $+($ Genotype + Error) $)$; and a model accounting for familiar relatedness $(K)$ [Phenotype $=S N P+$ Genotype + Error $]$ with Genotype random effects structured following a kinship matrix $K$. For each chromosome, a different kinship matrix was calculated where only the SNPs located on the other nine maize chromosomes were used to calculate the kinship matrix $[46,47]$.

The inspection of the inflation values for each model and the quantile-quantile (Q-Q) plots of the respective $P$-values allowed to informally assess to what extent the models accounted for genetic structure/relatedness among the inbred lines and therefore guarded against false discoveries. Models resulting in an inflation factor near 1 perform better, and quantile-quantile $(\mathrm{Q}-\mathrm{Q})$ plots should show few $P$-values that deviate from the expected uniform distribution that holds under the null hypothesis (i.e., no association). The observed $P$-values (on a $\log _{10}$ scale) of each SNP were plotted against their chromosomal positions so they produce Manhattan plots. A threshold of $-\log _{10}(P$-value $)=4$ was set to identify significant marker-trait associations. Given that the association panel used was not that large and looking at the background noise in the obtained Manhattan plots, the threshold of $-\log 10$ (P-value) $=4$ seemed reasonable not to avoid losing potentially interesting regions, at the expense of applying a more conservative type of correction such as Bonferroni. Examples of other maize studies with similar association panel sizes and number of markers using the same threshold are the works of Liu et al. $[48,49]$. The effect of the minor frequency SNP variant, reported in relation to the most frequent allele reference, was calculated.

\section{Post-GWAS procedures \\ Local linkage disequilibrium and candidate genes identification}

A local linkage disequilibrium (LD) study was performed to define chromosomal regions to search for candidate genes for the traits under analysis.
This procedure was done in two steps: In Step 1, the average intra-chromosomal LD was estimated as the squared correlation coefficient $\mathrm{r}^{2}$, after correcting for population structure using the principal component scores from Eigenanalysis, as implemented in Genstat software. For this calculation, the same subset of 1821 SNPs previously used for the genetic structure analysis was employed. LD decay was visualized per chromosome by plotting $\mathrm{r}^{2}$ against the physical mapping distance in Mb. A threshold for LD decay $\left(r^{2}=0.1\right)$ was used to estimate the average genetic distance for which markers were considered to be no longer correlated. In Step 2, a genomic window around each SNP location significantly associated with the traits analyzed was established by subtracting and adding the average genetic distance for LD decay $\left(\mathrm{r}^{2}>0.1\right)$, estimated in Step 1. All the SNP markers located within those windows were then used to estimate the local LD decay. At this point, a stricter threshold of $r^{2}=0.2$ was considered. The markers' positions flanking each local LD block were further used as query positions on the maize genome browser, via MaizeGDB (https://www.maizegdb.org/gbrowse/), to retrieve the list of candidate genes mapped within those genomic regions.

The genome sequence of the maize inbred line B73 (Zea mays B73 RefGen_v3) was used as the reference genome for candidate gene analyses [50]. The functional annotation of the genes under the identified genomic regions was retrieved via Phytozome ([51], Phytozome 11, version AGPv3 - Zea mays Ensembl-18) using the gene model identifier as the query. KEGG: Kyoto Encyclopedia of Genes and Genomes database [52] was used to retrieve information on the pathways where the candidate genes could be involved.

\section{Additional files}

\begin{abstract}
Additional file 1: Table S1. Pearson correlation coefficients among quality traits measured in wholemeal flour of 132 maize inbred lines. In Table S1 one can find the pairwise Pearson correlations coefficients between the 11 quality trait evaluated and the $P$-value of the 2-tailed test for each growing season. (DOCX $28 \mathrm{~kb}$ )
\end{abstract}

Additional file 2: Table S2. Estimated genetic correlations among quality traits measured in wholemeal flour of a collection of 132 maize inbred lines. In Table S2 one can find the estimated pairwise genetic correlation between the 11 quality trait evaluated for each growing season. (DOCX $22 \mathrm{~kb}$ )

Additional file 3: Table S3. Phenotypic values (range, and mean \pm standard deviation) for 11 quality traits measured in 132 maize inbred lines. In Table S3 one can find for the 11 quality trait evaluated the summary statistics on phenotypic data for each growing season and across growing seasons. (DOCX $24 \mathrm{~kb}$ )

Additional file 4: Table S4. Observed inflation factors for the models tested in genome-wide association (GWAS) analysis. In Table S4 one can find the inflation factors for each of the three different models that were tested to detect significant marker-trait associations: the naive model, a 
model accounting for population structure; and a model accounting for familiar relatedness. (DOCX $21 \mathrm{~kb}$ )

Additional file 5: Table S5. Significant SNP-trait associations from a genome-wide association study for 11 quality traits in wholemeal maize flour. In Table S5 one can find the genome-wide association study results for the 11 quality trait evaluated based on the information combining the phenotypic data obtained in two growing seasons with the genotypic data obtained from the Illumina MaizeSNP50 BeadChip array using 132 maize inbred lines. Additionally, Table S5 also encloses information on the genomic regions associated to quality-related traits previously identified by other authors. (XLSX $22 \mathrm{~kb}$ )

Additional file 6: Table S6. Percentage of associated SNPs with opposite effects on each traits value, and the range of phenotypic variance explained. In Additional file 6: Table 56 one can find the summarized information on the overall abundance of alleles increasing and decreasing the trait value in the inbred line collection; information on the range of phenotypic variance explained by significantly associated SNPs; information on the effect of rare allele explaining the largest phenotypic variance. (DOCX $23 \mathrm{~kb}$ )

Additional file 7: Table S7. Candidate genes underlying the genomic regions associated with 11 quality traits in wholemeal maize flour. In Additional file 7: Table $\mathrm{S7}$ one can find the information on the candidate gene annotations and information on its function. (XLSX $29 \mathrm{~kb}$ )

Additional file 8: Table S8. Maize inbred lines with available quality data, known pedigree, kernel color, and endosperm type. In Additional file 8: Table $\mathbf{8} 8$ one can find the list of 132 inbred lines that yielded sufficient kernels to proceed for quality analysis. The underlined inbred lines correspond to the lines derived entirely from Portuguese traditional maize populations, according to the Portuguese Plant Germplasm Bank records. (DOCX $34 \mathrm{~kb}$ )

Additional file 9: Table S9. Number of maize inbred lines grouped accordingly to their kernel color and endosperm type. In Additional file 9: Table 59 one can find the summary of the number of inbred lines with the different kernel colors and endosperm types. (DOCX 21 $\mathrm{kb})$

\section{Abbreviations}

BD: Breakdown viscosity; BD_SqRt: Breakdown viscosity, squared-root-transformed values; BLUEs: Best linear unbiased estimators; BLUPs: Best linear unbiased predictors; Fl: Fiber content; FT: Fat content; FT-NIR: Fourier Transform Near-Infrared Reflectance; FV: Final viscosity; GXE: Genotype-by-environment; GWAS: Genome-wide association study; LD: Linkage disequilibrium; MAF: Minor allele frequency; PCA: Principal component analysis; PR: Protein content; PSI: Particle Size Index; PV: Peak (or maximum viscosity); Q-Q: Quantile-quantile; QTL(s): Quantitative trait locus (loci); REML: Residual maximum likelihood; RVA: Rapid Visco Analyser; SB1: Setback from trough viscosity; SB2: Setback from peak viscosity; SIZE: Mean particle size; SIZEL: Mean particle size in lyophilized samples; SNP(s): Single nucleotide polymorphism(s); ST: Starch content; STL: Starch content in lyophilized samples; TV: Trough (or minimum) viscosity

\section{Acknowledgements}

The authors would like to acknowledge the National Portuguese Bank of Plant Germplasm (BPGV, Braga, Portugal) for providing the original seeds of the maize inbred lines.

\section{Funding}

The research leading to these results has received funding from the European Union's Seventh Framework Programme (FP7/2007-2013) and Horizon 2020 Framework Programme under grant agreements No 245058 and No 633571, respectively, and from the Fundação para a Ciência e a Tecnologia, through the research unit GREEN-IT (UID/Multi/04551/2013), and through the research project under the grant agreement no. PTDC/AGR-ALI/099285/2008. MLA and MCVP were supported by Fundação para a Ciência e a Tecnologia (SFRH/BD/ 77723/2011; FCT Investigator 2015 contract - IF/01337/2014, respectively).

\section{Availability of data and materials}

The datasets used during the current study are available from the corresponding author on reasonable request.

\section{Authors' contributions}

MLA performed the DNA isolation, the genotypic and phenotypic data analysis, the association mapping analysis and follow-up analysis, and drafted the manuscript. $\mathrm{BC}$ performed the quality data acquisition. $\mathrm{DG}, \mathrm{CB}$, and $\mathrm{MP}$ participated in the establishment and maintenance of the field trials and performed the controlled pollinations. PMM coordinated the field trials and participated in the manuscript revision. CMB coordinated and participated in the acquisition of quality data, and participated in the manuscript revision. MM and FvE participated in the molecular and phenotypic data processing, in the genome-wide association analysis, and participated in the manuscript revision. MCVP designed and coordinated the study and participated in the drafting and revising of the manuscript. All authors read and approved the final manuscript.

\section{Ethics approval and consent to participate}

Not applicable.

\section{Consent for publication}

Not applicable.

\section{Competing interests}

The authors declare that they have no competing interests.

\section{Publisher's Note}

Springer Nature remains neutral with regard to jurisdictional claims in published maps and institutional affiliations.

\section{Author details}

'Instituto de Tecnologia Química e Biológica António Xavier, Universidade Nova de Lisboa, Oeiras, Portugal. ${ }^{2}$ Instituto Nacional de Investigação Agrária e Veterinária, Oeiras, Portugal. ${ }^{3}$ Instituto Politécnico de Coimbra - Escola Superior Agrária, Coimbra, Portugal. ${ }^{4}$ Wageningen University \& Research, Wageningen, The Netherlands.

Received: 28 June 2018 Accepted: 19 March 2019

Published online: 02 April 2019

\section{References}

1. Ai Y, Jane J. Macronutrients in corn and human nutrition. Compr Rev Food Sci Food Saf. 2016;15:581-98 Available from: http://doi.wiley.com/10.1111/ 1541-4337.12192.

2. Muzhingi T, Palacios-Rojas N, Miranda A, Cabrera ML, Yeum K-J, Tang G. Genetic variation of carotenoids, vitamin $\mathrm{E}$ and phenolic compounds in Provitamin a biofortified maize. J Sci Food Agric. 2017;97:793-801 Available from: http://doi.wiley.com/10.1002/jsfa.7798.

3. Rodríguez VM, Soengas P, Landa A, Ordás A, Revilla P. Effects of selection for color intensity on antioxidant capacity in maize (Zea mays L.). Euphytica. 2013;193:339-45 Available from: http://link.springer.com/10.1007/s10681013-0924-0.

4. Nuss ET, Tanumihardjo SA. Maize: a paramount staple crop in the context of global nutrition. Compr Rev Food Sci Food Saf. 2010;9:417-36 Available from: http://doi.wiley.com/10.1111/j.1541-4337.2010.00117.x.

5. Wen W, Brotman Y, Willmitzer L, Yan J, Fernie AR. Broadening our portfolio in the genetic improvement of maize chemical composition. Trends Genet. 2016;32:459-69 Available from: https://doi.org/10.1016/j.tig.2016.05.003.

6. Rose DJ, Inglett GE, Liu SX. Utilisation of corn (Zea mays) bran and corn fiber in the production of food components. J Sci Food Agric. 2010;90:915-24 Available from: http://doi wiley.com/10.1002/jsfa.3915.

7. Wang S, Zheng M, Yu J, Wang S, Copeland L. Insights into the formation and structures of starch-protein-lipid complexes. J Agric Food Chem. 2017; 65:1960-6 Available from: http://pubs.acs.org/doi/10.1021/acs.jafc.6b05772.

8. Zhang G, Hamaker BR. A three component interaction among starch, protein, and free fatty acids revealed by pasting profiles. J Agric Food Chem. 2003:51:2797-800 Available from: http://pubs.acs.org/doi/abs/10 1021/jf0300341.

9. Hartings H, Fracassetti M, Motto M. Genetic enhancement of grain qualityrelated traits in maize. Transgenic plants - Adv limitations. Rijeka. Croatia: InTech; 2012. p. 191-218. Available from: http://www.intechopen.com/ books/transgenic-plants-advances-and-limitations/genetic-enhancement-ofgrain-quality-related-traits-in-maize. 
10. Wang T, Wang M, Hu S, Xiao Y, Tong H, Pan Q, et al. Genetic basis of maize kernel starch content revealed by high-density single nucleotide polymorphism markers in a recombinant inbred line population. BMC Plant Biol. 2015;15:288 Available from: https://doi.org/10.1186/s12870-015-0675-2.

11. Li H, Peng Z, Yang X, Wang W, Fu J, Wang J, et al. Genome-wide association study dissects the genetic architecture of oil biosynthesis in maize kernels. Nat Genet. 2013;45:43-50 Available from: https://doi.org/ 10.1038/ng.2484.

12. Li C, Huang $Y$, Huang $R$, Wu Y, Wang W. The genetic architecture of amylose biosynthesis in maize kernel. Plant Biotechnol J. 2018;16:688-95.

13. Cook JP, McMullen MD, Holland JB, Tian F, Bradbury P, Ross-lbarra J, et al. Genetic architecture of maize kernel composition in the nested association mapping and inbred association panels. Plant Physiol. 2012;158:824-34 Available from: http://www.plantphysiol.org/cgi/doi/10.1104/pp.111.185033.

14. Wilson LM, Whitt SR, Ibáñez AM, Rocheford TR, Goodman MM, Buckler ES. Dissection of maize kernel composition and starch production by candidate Gene Association. Plant Cell. 2004;16:2719-33 Available from: http://www. plantcell.org/cgi/doi/10.1105/tpc.104.025700.

15. Xu S, Yang Z, Zhang E, Jiang Y, Pan L, Chen Q, et al. Nucleotide diversity of maize ZmBT1 gene and Association with starch physicochemical properties. PLoS One. 2014;9:e103627 Available from: https://doi.org/10.1371/journal. pone. 0103627

16. Patto MCV, Satovic Z, Pêgo S, Fevereiro P. Assessing the genetic diversity of Portuguese maize germplasm using microsatellite markers. Euphytica. 2004; 137:63-72 Available from: http://link.springer.com/10.1023/B:EUPH. 0000040503.48448 .97$.

17. Vaz Patto MC, Moreira PM, Carvalho V, Pego S. Collecting maize (Zea mays L. convar. mays) with potential technological ability for bread making in Portugal. Genet Resour Crop Evol. 2007;54:1555-63 Available from: http:// link.springer.com/10.1007/s10722-006-9168-3.

18. Brites C, Trigo MJ, Santos C, Collar C, Rosell CM. Maize-based gluten-free bread: influence of processing parameters on sensory and instrumental quality. Food Bioprocess Technol. 2010;3:707-15 Available from: http://link. springer.com/10.1007/s11947-008-0108-4.

19. Alves ML, Brites C, Paulo M, Carbas B, Belo M, Mendes-Moreira PMR, et al. Setting up decision-making tools toward a quality-oriented participatory maize breeding program. Front Plant Sci. 2017:8:1-15 Available from: http:// journal.frontiersin.org/article/10.3389/fpls.2017.02203/full.

20. Paraginski RT, Vanier NL, Moomand K, de Oliveira M, Zavareze EDR, e Silva $\mathrm{RM}$, et al. Characteristics of starch isolated from maize as a function of grain storage temperature. Carbohydr Polym 2014;102:88-94. Available from: https://doi.org/10.1016/j.carbpol.2013.11.019

21. Carbas B, Vaz-Patto MC, Bronze MR, Bento-Da-Silva A, Trigo MJ, Brites C. Maize flour parameters that are related to the consumer perceived quality of 'broa' specialty bread. Food Sci Technol. 2016;36:259-67 Available from: https://doi.org/10.1590/1678-457X.6674.

22. de la Hera E, Talegón M, Caballero P, Gómez M. Influence of maize flour particle size on gluten-free breadmaking. J Sci Food Agric. 2013;93:924-32 Available from: http://doi.wiley.com/10.1002/jsfa.5826.

23. Fox G, Manley M. Hardness methods for testing maize kernels. J Agric Food Chem. 2009;57:5647-57 Available from: http:/pubs.acs.org/doi/abs/10.1021/jf900623w.

24. Chandrashekar A, Mazhar $\mathrm{H}$. The biochemical basis and implications of grain strength in Sorghum and maize. J Cereal Sci. 1999:30:193-207 Available from: http://linkinghub.elsevier.com/retrieve/pii/S0733521099902643.

25. Wang G, Wang G, Wang J, Du Y, Yao D, Shuai B, et al. Comprehensive proteomic analysis of developing protein bodies in maize (Zea mays) endosperm provides novel insights into its biogenesis. J Exp Bot. 2016; 67:6323-35 Available from: https://academic.oup.com/jxb/article-lookup/ doi/10.1093/jxb/erw396.

26. Karn A, Gillman JD, Flint-Garcia SA. Genetic analysis of teosinte alleles for kernel composition traits in maize. G3 genes/genomes/genetics. Genetics. 2017;7:115764 Available from: http://g3journal.org/lookup/doi/10.1534/g3.117.039529.

27. Chen X, Feng F, Qi W, Xu L, Yao D, Wang Q, et al. Dek35 encodes a PPR protein that affects cis -splicing of mitochondrial nad4 intron 1 and seed development in maize. Mol Plant. 2017;10:427-41 Available from: https:// doi.org/10.1016/j.molp.2016.08.008.

28. Gao M, Wanat J, Stinard PS, James MG, Myers AM. Characterization of dull1, a maize gene coding for a novel starch synthase. Plant Cell Online. 1998;10: 399-412 Available from: http://www.plantcell.org/cgi/doi/10.1105/tpc.10.3.399.

29. Wu JD, Jiang CP, Zhu HS, Jiang HY, Cheng BJ, Zhu SW. Cloning and functional analysis of the promoter of a maize starch synthase III gene
(ZmDULL 1). Genet Mol Res. 2015;14:5468-79 Available from: http://www. ncbi.nlm.nih.gov/pubmed/26125743.

30. Zheng P, Allen WB, Roesler K, Williams ME, Zhang S, Li J, et al. A phenylalanine in DGAT is a key determinant of oil content and composition in maize. Nat Genet. 2008;40:367-72 Available from: http://www.nature.com/articles/ng.85.

31. Yang X, Yan J, Shah T, Warburton ML, Li Q, Li L, et al. Genetic analysis and characterization of a new maize association mapping panel for quantitative trait loci dissection. Theor Appl Genet. 2010;121:417-31 Available from: http://link.springer.com/10.1007/s00122-010-1320-y.

32. Korte A, Farlow A. The advantages and limitations of trait analysis with GWAS: a review. Plant Methods. 2013;9:29 Available from: http://plantmethods. biomedcentral.com/articles/10.1186/1746-4811-9-29.

33. Cantor RM, Lange K, Sinsheimer JS. Prioritizing GWAS results: a review of statistical methods and recommendations for their application. Am J Hum Genet. 2010;86:6-22 Available from: https://doi.org/10.1016/j.ajhg.2009.11.017.

34. Huang $X$, Han B. Natural variations and genome-wide association studies in crop plants. Annu Rev Plant Biol. 2014;65:531-51 Available from: http:// www.annualreviews.org/doi/10.1146/annurev-arplant-050213-035715.

35. Mendes-Moreira P, Alves ML, Satovic Z, dos Santos JP, Santos JN, Souza JC, et al. Genetic architecture of ear fasciation in maize (Zea mays) under QTL scrutiny. PLoS One 2015;10:e0124543. Available from: http://dx.plos.org/10. 1371/journal.pone.0124543.

36. AACC. Particle Size of Wheat Flour by Laser Instrument. AACC Int Approv Methods. 11th ed. St. Paul: AACC International; 2009. p. 10-1. Available from: http://methods.aaccnet.org/summaries/55-40-01.aspx.

37. Almeida-Dominguez HD, Suhendro EL, Rooney LW. Factors affecting rapid Visco Analyser curves for the determination of maize kernel hardness. J Cereal Sci. 1997;25:93-102 Available from: http://linkinghub.elsevier.com/ retrieve/pii/S0733521096900727.

38. Murray D, Payne R, Zhang Z. Breeding view, a visual tool for running analytical pipelines: user guide: VSN International Ltd.; Hemel Hempstead, UK; 2015. Available from: https:/www.integratedbreeding.net/attachment/1075/ BreedingViewGuide.pdf. Accessed 28 June 2018.

39. Oakey H, Arunas V, Pitchford W, Cullis B, Kuchel H. Joint modeling of additive and non-additive genetic line effects in single field trials. Theor Appl Genet. 2006;113:809-19.

40. Payne R, Murray D, Harding S. GenStat for Windows (18th Edition) Introduction. Hemel Hempstead: VSN International Ltd.; 2015. Available from: http://cdn.vsni. co.uk/downloads/genstat/release18/doc/Commandlntro.pdf.

41. Saghai-Maroof MA, Soliman KM, Jorgensen RA, Allard RW. Ribosomal DNA spacer-length polymorphisms in barley: mendelian inheritance, chromosomal location, and population dynamics. Proc Natl Acad Sci. 1984;81:8014-8 Available from: http://www.pnas.org/cgi/doi/10.1073/pnas.81.24.8014.

42. Rether B, Delmas $G$, Laouedj A. Isolation of polysaccharide-free DNA from plants. Plant Mol Biol Report. 1993;11:333-7 Available from: http://link. springer.com/10.1007/BF02905335.

43. Ganal MW, Durstewitz G, Polley A, Bérard A, Buckler ES, Charcosset A, et al. A large maize (Zea mays L.) SNP genotyping Array: development and germplasm genotyping, and genetic mapping to compare with the B73 reference genome. PLoS One. 2011;6:e28334 Available from: http://dx.plos. org/10.1371/journal.pone.0028334.

44. Lawrence CJ, Harper LC, Schaeffer ML, Sen TZ, Seigfried TE, Campbell DA. MaizeGDB: the maize model organism database for basic, translational, and applied research. Int J Plant Genomics. 2008;2008:1-10 Available from: http://www.hindawi.com/journals/ijpg/2008/496957/.

45. Malosetti $M$, van der Linden CG, Vosman B, van Eeuwijk FA. A mixed-model approach to association mapping using pedigree information with an illustration of resistance to Phytophthora infestans in potato. Genetics. 2007; 175:879-89 Available from: http://www.genetics.org/cgi/doi/10.1534/ genetics.105.054932.

46. Listgarten J, Lippert C, Kadie CM, Davidson RI, Eskin E, Heckerman D. Improved linear mixed models for genome-wide association studies. Nat Methods. 2012;9:525-6 Available from: https://doi.org/10.1038/nmeth.2037.

47. Rincent R, Moreau L, Monod H, Kuhn E, Melchinger AE, Malvar RA, et al. Recovering power in association mapping panels with variable levels of linkage disequilibrium. Genetics. 2014;197:375-87 Available from: http:// www.genetics.org/lookup/doi/10.1534/genetics.113.159731.

48. Liu N, Xue Y, Guo Z, Li W, Tang J. Genome-wide association study identifies candidate genes for starch content regulation in maize kernels. Front Plant Sci. 2016;7:1-8 Available from: http://journal.frontiersin.org/Article/10.3389/ fpls.2016.01046/abstract. 
49. Liu N, Zhang Z, Xue Y, Meng S, Huang Y, Li W. Identification of quantitative trait loci and candidate genes for maize starch granule size through association mapping. Sci Rep. 2018;8:1-11.

50. Schnable PS, Ware D, Fulton RS, Stein JC, Wei F, Pasternak S, et al. The B73 maize genome: complexity, diversity, and dynamics. Science. 2009;326: 1112-5 Available from: http://www.sciencemag.org/cgi/doi/10.1126/ science.1178534.

51. Goodstein DM, Shu S, Howson R, Neupane R, Hayes RD, Fazo J, et al. Phytozome: a comparative platform for green plant genomics. Nucleic Acids Res. 2012;40:D1178-86 Available from: https://academic.oup.com/ nar/article-lookup/doi/10.1093/nar/gkr944.

52. Kanehisa M, Goto SKEGG. Kyoto encyclopedia of genes and genomes. Nucleic Acids Res. 2000;28:27-30 Available from: https://academic.oup. com/nar/article-lookup/doi/10.1093/nar/27.1.29.

Ready to submit your research? Choose BMC and benefit from:

- fast, convenient online submission

- thorough peer review by experienced researchers in your field

- rapid publication on acceptance

- support for research data, including large and complex data types

- gold Open Access which fosters wider collaboration and increased citations

- maximum visibility for your research: over $100 \mathrm{M}$ website views per year

At $\mathrm{BMC}$, research is always in progress.

Learn more biomedcentral.com/submissions 